\title{
INCLUSION OF AERODYNAMIC EFFECTS IN MULTIBODY DYNAMICS
}

\author{
BP Minaker ${ }^{\dagger} \mathrm{PhD}$ PEng, M Sun, J Defoe PhD \\ Department of Mechanical, Automotive, \& Materials Engineering \\ University of Windsor \\ Windsor, Ontario, Canada \\ bminaker@uwindsor.ca
}

\begin{abstract}
The Equations of Motion (EoM) software, developed by University of Windsor Vehicle Dynamics and Control Research Group, can be used to generate linear or linearized equations of motion for mechanical systems, and is particularly well suited to vehicle dynamics. This paper describes an effort to extend its capability to include the effects of wings on the motion of multibody systems.
\end{abstract}

Keywords- multibody dynamics; aerodynamics; equations of motion; stability; eigenvalues; open source software

\section{INTRODUCTION}

This paper discusses the Equations of Motion (EoM) multibody dynamics software developed by University of Windsor Vehicle Dynamics and Control Research Group, and an attempt to extend its capability to include the effects of wings on the motion of multibody systems.

The EoM software can be used to generate linear or linearized equations of motion for multibody mechanical systems. The software is entirely open source and runs within the MATLAB ${ }^{\circledR}$ framework, or in the syntax compatible open source alternative, Octave. It has also recently been ported to Julia, a new open source high performance programming language optimized for numerical computing. It is freely available online at the software hosting site www.github.com.

The input of the software is a simple function file describing the system in question, and the output is the state space form of the equations, and optionally, a report tabulating the results of a linear analysis in a .pdf format, and animations of the mode shapes that can be viewed using any virtual reality modelling language (VRML) viewer.

When analyzing a multibody system, EoM will first read the information from the input data, and build the necessary stiffness and constraint Jacobian matrices required to find all the preload and constraint forces. Once these are known, the stiffness matrices are updated with the tangent stiffness terms. The kinematic differential equations relating position and velocity

\footnotetext{
$\dagger$ corresponding author
}

are then combined with the Newton-Euler equations of motion, reduced to a minimal coordinate set, and cast in the following form:

$$
\left[\begin{array}{ll}
\mathbf{E} & \mathbf{0} \\
\mathbf{0} & \mathbf{I}
\end{array}\right]\left\{\begin{array}{l}
\dot{x} \\
y
\end{array}\right\}=\left[\begin{array}{ll}
\mathbf{A} & \mathbf{B} \\
\mathbf{C} & \mathbf{D}
\end{array}\right]\left\{\begin{array}{l}
\boldsymbol{x} \\
\boldsymbol{u}
\end{array}\right\}
$$

A careful examination will show that this system, known as the descriptor form of the state space, can be further reduced to standard state space form, if the $\mathbf{E}$ matrix is non-singular. If it is singular, then the system becomes a set of differentialalgebraic equations. Nevertheless, a singular value decomposition approach can be used to reduce the system to an equivalent lower dimensional standard state space form. From this state matrix form, EoM will perform a number of linear analyses, e.g., calculate the eigenvalue of each mode to determine the stability, compute the frequency response between any inputs or outputs that have been defined, and determine the minimal realization (the minimum dimensional system with equivalent input-output behaviour).

\section{EXTENSION TO INCLUDE AERODYNAMIC EFFECTS}

The intention of the work described in this paper is to extend the capabilities of the EoM software to include aerodynamic effects. The EoM software is primarily used to model vehicle dynamics; there are many cases where aerodynamic effects could have significant influence on vehicle behaviour. The addition of aerodynamic items to EoM would enable automated construction of multibody dynamic models of a number of systems, e.g, a vehicle ride (bounce-pitch) model with front and rear wings, a motorcycle with an aerodynamic fairing, or an aircraft with discrete flexible model elements (e.g. a torsionally flexible fuselage). More elaborate examples might include an aircraft during lift-off or landing, where both the tires and the aero surfaces are contributing to the motion, or an aircraft towing a second aircraft with a cable, as is commonly used when launching gliders.

\section{A. Basic wing example}

To illustrate the inclusion of aero effects on a dynamic vibrating system, consider the equations of motion for the 
spring mass system, with a single wing attached, as depicted in Fig. 1. The development begins with the breakdown of the net aerodynamic force into lift and drag. The equations for lift force $L$ and drag force $D$ are written as a function of the dynamic pressure $q$, where $\rho$ represents the air density, $V$ the airstream velocity, and $S$ the wing area.

$$
\begin{aligned}
q & =\frac{1}{2} \rho V^{2} \\
L & =q S C_{\mathrm{L}} \\
D & =q S C_{\mathrm{D}}
\end{aligned}
$$

The nondimensional coefficients of lift and drag are $C_{\mathrm{L}}$ and $C_{\mathrm{D}}$, respectively.

Note that the lift and drag forces are assumed to remain parallel and perpendicular to the airflow direction respectively, measured in a reference frame that moves with the wing. If the wing is moving vertically downward, the airflow appears to have an upward velocity component, quantified by the angle of attack. The angle of attack can be written in terms of the vertical speed of the wing, and simplified by assuming small angles.

$$
\alpha \approx \alpha_{0}-\frac{\dot{z}}{V}
$$

In effect, the directions of the aero forces as defined above change relative to the ground fixed reference frame. Taking the vertical component of the aero forces gives:

$$
Z=L \cos \left(\alpha-\alpha_{0}\right)+D \sin \left(\alpha-\alpha_{0}\right)
$$

To linearize, a truncated Taylor series is used.

$$
Z \approx Z_{0}+\left.\frac{\partial Z}{\partial \alpha}\right|_{\alpha=\alpha_{0}}\left(\alpha-\alpha_{0}\right)
$$

The lift and drag coefficients are assumed to be functions of the angle of attack.

$$
\begin{array}{r}
\frac{\partial Z}{\partial \alpha}=\frac{\partial L}{\partial \alpha} \cos \left(\alpha-\alpha_{0}\right)-L \sin \left(\alpha-\alpha_{0}\right) \\
+\frac{\partial D}{\partial \alpha} \sin \left(\alpha-\alpha_{0}\right)+D \cos \left(\alpha-\alpha_{0}\right) \\
\left.\frac{\partial Z}{\partial \alpha}\right|_{\alpha=\alpha_{0}}=\frac{\partial L}{\partial \alpha}+D=q S\left(\frac{\partial c_{\mathrm{L}}}{\partial \alpha}+c_{\mathrm{D}}\right)
\end{array}
$$

As a result:

$$
Z=Z_{0}+q S\left(\frac{\partial c_{\mathrm{L}}}{\partial \alpha}+c_{\mathrm{D}}\right)\left(\alpha-\alpha_{0}\right)
$$

Noting that $\alpha-\alpha_{0}=-\dot{z} / V$

$$
Z=Z_{0}-\frac{q S}{V}\left(\frac{\partial c_{\mathrm{L}}}{\partial \alpha}+c_{\mathrm{D}}\right) \dot{z}
$$

Combining the sum of the aerodynamic and spring forces, and equating to mass times acceleration gives the familiar second order linear differential equation of motion. Note that the deflection $z$ is measured from equilibrium, where any static lift forces $Z_{0}$ are offset by the spring preload $k z_{0}$, (weight force is neglected, but would have no effect in this example).

$$
m \ddot{z}=Z-k\left(z+z_{0}\right)=Z_{0}-\frac{1}{V} \frac{\partial Z}{\partial \alpha} \dot{z}-k\left(z+z_{0}\right)
$$

Simplifying:

$$
m \ddot{z}+\frac{q S}{V}\left(c_{L \alpha}+c_{\mathrm{D}}\right) \dot{z}+k z=0
$$

The dynamic pressure $q$ is parabolic in $V$, so the wing behaves effectively as a damper whose coefficient increases linearly with airspeed. This is noteworthy in a vehicle dynamics context, as it is in contrast to a typical slip-based tire model, where the tire is effectively a damper whose coefficient varies as the inverse of forward speed.

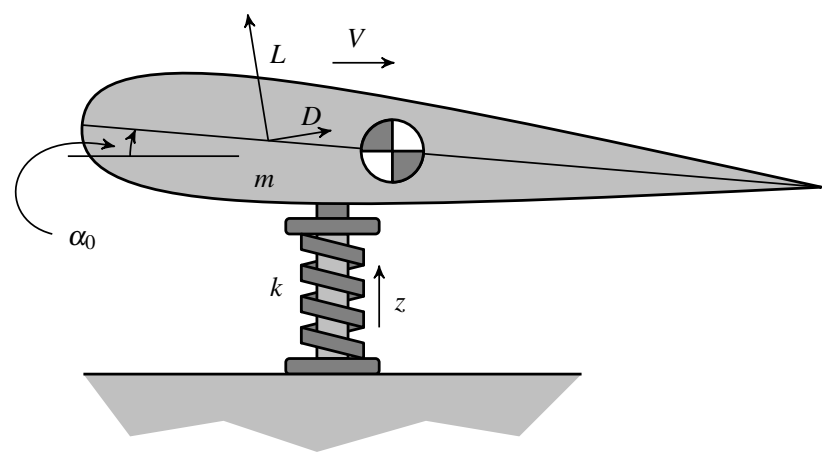

Figure 1. Spring mass wing model. Note that the lift and drag forces are assumed to remain parallel and perpendicular to the airflow direction respectively, measured in a reference frame that moves with the wing.

While the example illustrates the development of the equation of motion for a simple system, in fully three dimensional motion, the effective damping term becomes a full $6 \times 6$ matrix, where the three forces and three moments are written as linear functions of linear and angular speed. A typical vehicle based coordinate system is used, where the $\mathrm{x}$ axis is the vehicle's direction of travel, and the $\mathrm{z}$ axis is vertical. Symmetry in the $\mathrm{xz}$ plane is assumed; the effect is that fully half of the 36 damping terms go to zero. In this notation, the components of force in the three coordinate axes are $X, Y, Z$, while $L, M, N$ are the corresponding moments. The linear and angular velocities are represented as $u, v, w$, and $p, q, r$, respectively. The wingspan is denoted as $b$, and $c$ is the chord (i.e., $S=b c$ for a rectangular planform wing). These $6 \times 6$ matrices can be easily incorporated into the EoM generated equations of motion.

The full equation is written as: 


$$
\left\{\begin{array}{c}
X \\
Y \\
Z \\
L / b \\
M / c \\
N / b
\end{array}\right\}=-\frac{q S}{V}\left[\begin{array}{cccccc}
C_{X u} & 0 & C_{X w} & 0 & C_{X q} & 0 \\
0 & C_{Y v} & 0 & C_{Y p} & 0 & C_{Y r} \\
C_{Z u} & 0 & C_{Z w} & 0 & C_{Z q} & 0 \\
0 & C_{L v} & 0 & C_{L p} & 0 & C_{L r} \\
C_{M u} & 0 & C_{M w} & 0 & C_{M q} & 0 \\
0 & C_{N v} & 0 & C_{N p} & 0 & C_{N r}
\end{array}\right]\left\{\begin{array}{c}
u \\
v \\
w \\
\frac{b}{2} p \\
\frac{c}{2} q \\
\frac{b}{2} r
\end{array}\right\}
$$

or:

$$
\left\{\begin{array}{c}
X \\
Y \\
Z \\
L \\
M \\
N
\end{array}\right\}=[\mathbf{L}]\left\{\begin{array}{c}
u \\
v \\
w \\
p \\
q \\
r
\end{array}\right\}
$$

where:

$$
\mathbf{L}=-\frac{q S}{V}\left[\begin{array}{cccccc}
C_{X u} & 0 & C_{X w} & 0 & \frac{1}{2} c C_{X q} & 0 \\
0 & C_{Y v} & 0 & \frac{1}{2} b C_{Y p} & 0 & \frac{1}{2} b C_{Y r} \\
C_{Z u} & 0 & C_{Z w} & 0 & \frac{1}{2} c C_{Z q} & 0 \\
0 & b C_{L v} & 0 & \frac{1}{2} b^{2} C_{L p} & 0 & \frac{1}{2} b^{2} C_{L r} \\
c C_{M u} & 0 & c C_{M w} & 0 & \frac{1}{2} c^{2} C_{M q} & 0 \\
0 & b C_{N v} & 0 & \frac{1}{2} b^{2} C_{N p} & 0 & \frac{1}{2} b^{2} C_{N r}
\end{array}\right]
$$

\section{B. EoM system elements}

In EoM, each component of a system is defined as an 'item'. The most basic type of item is a 'body', which defines a rigid body. The other types of items define either rigid or flexible connectors that are attached to one or more rigid bodies. All the available types of items are listed in Table I.

TABLE I. Types of items in EoM

\begin{tabular}{cl}
\hline Type of item & Definition \\
\hline body & $\begin{array}{l}\text { a rigid body } \\
\text { a two point elastic spring, with linear or torsional stiffness } \\
\text { and damping, non-zero free length }\end{array}$ \\
link & $\begin{array}{l}\text { a two point massless rigid link } \\
\text { a generic point constraint with a variable number of } \\
\text { constraint forces and moments }\end{array}$ \\
flex_point & $\begin{array}{l}\text { a point spring with translational and/or rotational } \\
\text { stiffness and damping } \\
\text { nh_point }\end{array}$ \\
$\begin{array}{l}\text { a non-holonomic constraint to prevent velocity but not } \\
\text { displacement } \\
\text { a zero mass beam spring with bi-directional bending } \\
\text { and shear stiffness } \\
\text { load }\end{array}$ & $\begin{array}{l}\text { constant forces or moments applied to the system } \\
\text { applied force or moment, proportional to an input signal } \\
\text { used to measure displacement, velocity, or acceleration }\end{array}$ \\
sensor &
\end{tabular}

A typical vehicle model in EoM will consist of rigid bodies, both rigid and flexible connectors, and other items like static loads (to include the effect of preloads), actuators to apply time varying forces, and sensors to measure the resulting motion. Joints constrain bodies together while allowing certain degrees of freedom; ball joints (spherical joints) and hinges (revolute joints) are both typical. Both are modelled in EoM as 'rigid_point' type items, where the appropriate number of constraints can be specified in each case, along with an axis to define the associated directions. Suspension springs and shock absorbers are modelled as uni-directional two-point springs, and tires as 'flex_point' items, i.e., springs with zero free length.

As the aerodynamic forces described above are expressed using a linear dependency on the velocities, they are included as a special case of the 'flex_point' item, where the full $6 \times 6$ matrix is supplied, as opposed to a typical point spring damper where a single coefficient and a direction vector suffice. It is noteworthy that the terms generated by traditional mechanical damper will always result in a symmetric damping matrix. Because the wing behaviour requires a potentially asymmetric matrix, there is no possible combination of dampers that would provide an equivalent motion response. In order to properly capture the wing behaviour, the full matrix must be supplied.

\section{Formulation of THE EQUATIONS OF MOtion}

The linearized equations of motion of the model are automatically generated by EoM. A detailed description of the method is given in Minaker and Rieveley[1], and expanded in Minaker[2]. The set of differential equations are written as

$$
\left[\begin{array}{ccc}
\mathbf{I} & \mathbf{0} & \mathbf{0} \\
\mathbf{0} & \mathbf{M} & -\mathbf{G} \\
\mathbf{0} & \mathbf{0} & \mathbf{0}
\end{array}\right]\left\{\begin{array}{l}
\dot{p} \\
\dot{w} \\
\dot{\boldsymbol{u}}
\end{array}\right\}+\left[\begin{array}{ccc}
\mathbf{V} & -\mathbf{I} & \mathbf{0} \\
\mathbf{K} & \mathbf{L} & -\mathbf{F} \\
\mathbf{0} & \mathbf{0} & \mathbf{I}
\end{array}\right]\left\{\begin{array}{l}
p \\
\boldsymbol{w} \\
\boldsymbol{u}
\end{array}\right\}=\left\{\begin{array}{c}
\mathbf{0} \\
\boldsymbol{f}_{\mathrm{c}} \\
\boldsymbol{u}
\end{array}\right\}
$$

where the mass matrix $\mathbf{M}$ results from the Newton-Euler equations, and is tri-diagonal as is typical. The stiffness matrix $\mathbf{K}$ is the sum of terms resulting from deflection of elastic elements, and additional tangent stiffness matrix terms resulting from preload in the connectors. The $\mathbf{L}$ matrix contains the traditional viscous damping matrix, plus terms due to the inertia forces, i.e., centripetal forces and gyroscopic moments. It is in this $\mathbf{L}$ matrix that the aero terms are included. The $\mathbf{V}$ matrix results from the linearization of the kinematic differential equations, and is nonzero only in the event of a non-zero velocity reference condition.

The $\mathbf{F}$ and $\mathbf{G}$ matrices are used to allow the inclusion of rate dependency in the input forces, i.e., the applied forces are written as

$$
\boldsymbol{f}_{\mathrm{a}}=\mathbf{F} \boldsymbol{u}+\mathbf{G} \dot{u}
$$

where $\boldsymbol{u}$ is the input. The $\boldsymbol{p}$ vector represents the global locations and small angle orientations, and the $\boldsymbol{w}$ vector represents the body fixed linear and angular velocities. The constraint forces $f_{\mathrm{c}}$ are still present in the intermediate formulation, but are eliminated through a coordinate reduction process. The linearized constraint equations are written as

$$
\left[\begin{array}{ccc}
\mathbf{J}_{\mathrm{h}} & \mathbf{0} & \mathbf{0} \\
-\mathbf{J}_{\mathrm{h}} \mathbf{V} & \mathbf{J}_{\mathrm{h}} & \mathbf{0} \\
\mathbf{0} & \mathbf{J}_{\mathrm{nh}} & \mathbf{0}
\end{array}\right]\left[\begin{array}{cc}
\dot{\boldsymbol{p}} & \boldsymbol{p} \\
\dot{\boldsymbol{w}} & \boldsymbol{w} \\
\dot{\boldsymbol{u}} & \boldsymbol{u}
\end{array}\right]=\left[\begin{array}{cc}
\mathbf{0} & \mathbf{0} \\
\mathbf{0} & \mathbf{0} \\
\mathbf{0} & \mathbf{0}
\end{array}\right]
$$

where the $\mathbf{J}_{\mathrm{h}}$ and $\mathbf{J}_{\mathrm{nh}}$ matrices represent holonomic and nonholonomic constraint equations, respectively. An orthogonal 
complement of the Jacobian is used to reduce the number of coordinates.

\section{WING ANALYSIS}

In order to generate the wing properties used to fill the associated damping matrix, a numerical approach is required. Luckily, a well-developed solution already exists.

\section{A. Athena Vortex Lattice}

Athena Vortex Lattice (AVL) is an open source software tool developed at MIT by Mark Drela and Harold Youngren[3]. It has numerous capabilities related to the flight-dynamic analysis of rigid aircraft of arbitrary configuration. While AVL can already produce a linearized aerodynamic model to predict aircraft stability, its models are limited to treating the aircraft as a single rigid body.

However, individual lifting and control surfaces can also be modelled in AVL (rather than full aircraft), and the full aerodynamic properties can be output in a format suitable for inclusion in other codes, such as EoM.

The relevant functionality of AVL for this paper is its ability to produce stability derivatives (the elements of the system matrix relating state derivatives to the state values) at any flight condition. By definition, vortex lattice codes work in three dimensions; it is not possible to carry out two-dimensional analysis using AVL. AVL can be expected to produce accurate results when the lifting surfaces are thin and angles of attack (and sideslip) are small ( $\ll 1 \mathrm{rad}$ ). In a vortex lattice code, the surfaces and the wakes that trail them downstream are represented with vortex sheets of infinitesimal thickness. By discretizing the aerodynamic surface(s) of interest with horseshoe vortices distributed along the span and chord, a numerical solution for the aerodynamic forces and moments along the surface(s) can be obtained. A full description of vortex lattice methods is beyond the scope of this paper, but see for example Lan[4], Lamar and Gloss[5], and Miranda, Elliot, and Baker[6] for the foundational work that underpins AVL. An important limitation of AVL is the assumption of quasi-steady flow[3]. This means that oscillatory motions must be sufficiently slow so that the period of oscillation is long compared to the time it takes for the flow to travel the length of the wing chord. This can be quantified using the reduced frequency $\beta$, where

$$
\beta=\frac{\omega c}{V} \ll 1
$$

For typical aircraft in flight this is almost always true, but in alternative applications of AVL for use in EoM such as ground vehicles, this condition should be checked. Finally, the angular velocities (roll, pitch, and yaw rates) must be slow enough to ensure that small angle assumptions continue to hold.
Quantitatively, this means:

$$
\begin{aligned}
& |p b / 2 V|<0.10 \\
& |q c / 2 V|<0.03 \\
& |r b / 2 V|<0.25
\end{aligned}
$$

Again, while this is normally not an issue for aircraft, if AVL analysis is used for other applications, these should be checked to ensure solution validity.

\section{EXAMPLE PROBLEM}

In order to demonstrate the capabilities of the combined EoM/AVL software tool, a test model was developed. It is an expansion of the swivel wheel problem, as presented by Schwab and Meijaard[7], and before them by Den Hartog[8]. The model is used to predict the shimmy phenomenon, as sometimes observed in aircraft nose wheel landing gear, or more commonly on the front wheels of a grocery cart. The model consists of a single rigid body, constrained to planar motion, and travelling at a fixed forward speed. The motion of the body is reacted by a lateral spring placed at the front, to represent the lateral stiffness of the wheel carrier or support, and a no-slip tire (modelled as a nonholonomic constraint) at the rear. The wheel shimmy problem is noteworthy, as it can predict unstable behaviour. The response is strongly dependent on the location of the mass centre; when it is near the midway point between the tire and the lateral spring, the system is stable, while locations near either end result in an unstable motion. The instability is oscillatory; the amplitude grows with time.

In this example, the shimmy problem is repeated with an aerodynamic surface is added to the model. A symmetric generic thin surface model is used for the airfoil. The reference point of the airfoil is taken at one quarter of the chord from the leading edge. The wing is fixed such that its reference point is located at the front of the body at the same location where the lateral stiffness acts. It is noteworthy that shifting the longitudinal location of the wing does have an effect on its influence. As the wing is moved closer to the zero-slip tire, its angle of attack is reduced. In fact, the tire constraint forces the angle of attack to be zero at that point, minimizing the influence of the wing on the overall motion. A schematic diagram of the problem is shown in Figure 2.

In order to establish a baseline result, the model is run first with no wing present. The default properties of the mechanical system are: $m=5.0 \mathrm{~kg}, l=1.0 \mathrm{~m}, I_{\mathrm{z}}=1.05 \mathrm{~kg} \mathrm{~m}^{2}, k=1.5 \mathrm{~N} / \mathrm{m}$, and $u=10.0 \mathrm{~m} / \mathrm{s}$. The location of the mass centre is varied from the front to the rear of the model. The results are then repeated with the wing present. The resulting aerodynamic properties are: $L_{2,2}=7.59895 \mathrm{Ns} / \mathrm{m}, L_{2,6}=-2.05293 \mathrm{Ns} / \mathrm{rad}$, $L_{6,2}=0.153319 \mathrm{Ns}, L_{6,6}=0.224167 \mathrm{Nms} / \mathrm{rad}, b=1.0 \mathrm{~m}$, and $c=0.5 \mathrm{~m}$. 


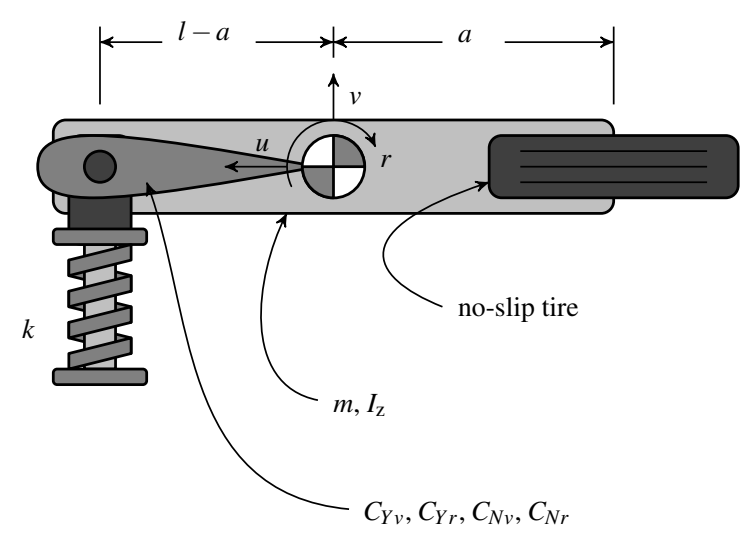

Figure 2. Swivel wheel example problem, with added aerodynamic effect. The swivel wheel exhibits the shimmy phenomenon, with potential instability, as a function of the location of the mass centre.

\section{A. Results}

Once the EoM software has generated the equations of motion, a number of linear analyses are automatically conducted. By analyzing the eigenvalues, the stability and natural frequencies of the motion can be found. The resulting eigenvalues govern the form of the motion. The eigenvalue can be expressed in the form of $s=a \pm b$ i, i.e., it is frequently a complex number. If the real part is positive, the motion will be unstable; if the real part is negative, the motion is stable as it gradually approaches zero with time. The imaginary part controls the oscillatory frequency of the motion. If the imaginary part is equal to zero, there will be no oscillation in the unforced motion.

The results are plotted in Figures 3 and 4. Figure 3 shows both eigenvalues. Note that the slow eigenvalue is complex, while the fast eigenvalue, i.e., the more negative, is real. Figure 4 shows the real part of the slow eigenvalue, which is unstable in some cases, with a much tighter zoom, to better illustrate the zero crossings. Note that if the centre of mass is located in the range $0.3 l<a<0.7 l$, then the system without the wing is stable. When the wing is added, the stability range increases to $0.12 l<a<0.88 l$.

\section{CONCLUSIONS}

The paper outlines the development of an open source multibody code for the analysis of the dynamics of mechanical systems, including aerodynamic effects. An example illustrates how mechanical system behaviour can be influenced by the addition of aerodynamic effects. The example shown is relatively simple in scope when compared to the type of problems that could be analyzed using the combined EoM and AVL software, but serves to illustrate the concept. Future work will expand this approach to more extensive applications, such as ground vehicles with multiple aerodynamic surfaces or flexible aircraft.

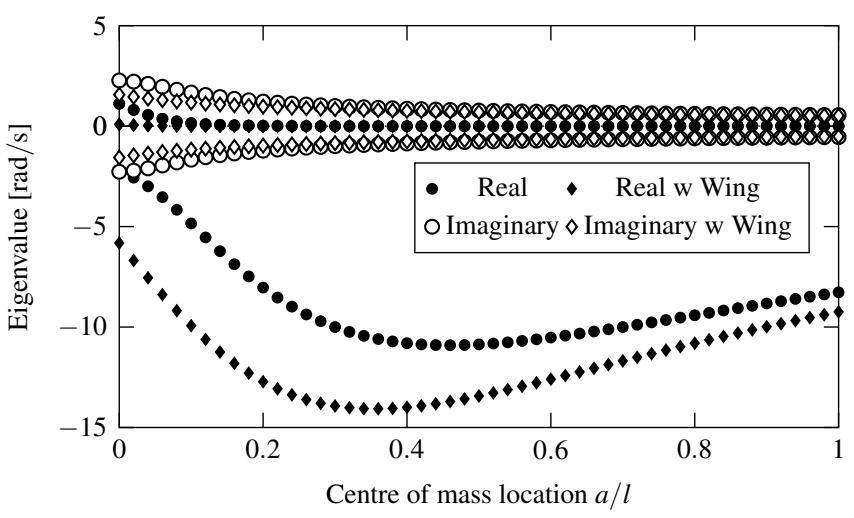

Figure 3. Eigenvalues vs centre of mass location. The effect of the addition of the wing on the eigenvalues is a significant increase in stability, and decrease in the shimmy frequency in the cases where the centre of mass is shifted rearward.

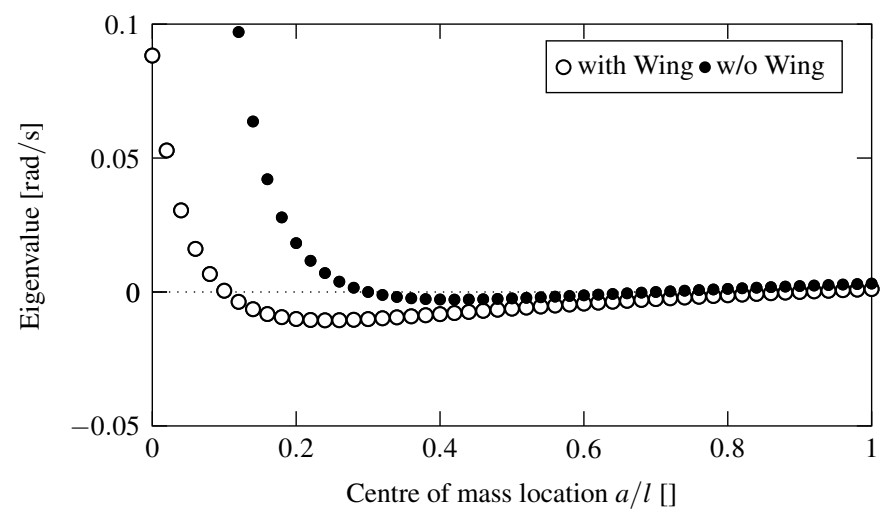

Figure 4. Slow eigenvalues vs centre of mass location, zoomed. Note that if the centre of mass is located in the range $0.3 l<a<0.7 l$ then the system without the wing is stable. When the wing is added the range is expanded.

\section{REFERENCES}

[1] B. Minaker and R. Rieveley, "Automatic generation of the nonholonomic equations of motion for vehicle stability analysis", Vehicle Systems Dynamics, vol. 48 (9), pp. 1043-1063, 2010

[2] B.P. Minaker, "The tangent stiffness matrix in rigid multibody vehicle dynamics", Mathematical and Computer Modelling of Dynamical Systems, doi:10.1080/13873954.2014.953549, 2014

[3] M. Drela and H. Youngren, "Athena vortex lattice", http://web.mit.edu/drela/Public/web/avl, January 10, 2018.

[4] C.E. Lan, "A Quasi-Vortex-Lattice Method in Thin Wing Theory", Journal of Aircraft, vol. 11 (9), pp. 518-527, 1974

[5] J.E. Lamar and B.B. Gloss, "Subsonic aerodynamic characteristics of interacting lifting surfaces with separated flow around sharp edges predicted by a vortex-lattice method", NASA Technical Report NASA-TN-D-7921, 1975

[6] L.R. Miranda, R.D. Elliot, and W.M. Baker, "A generalized vortex lattice method for subsonic and supersonic flow applications", NASA Technical Report NASA-CR-2865, 1977

[7] A.L. Schwab and J.P. Meijaard, "Dynamics of Flexible Multibody Systems with Non-Holonomic Constraints: A Finite Element Approach”, Multibody System Dynamics 10, pp. 107-123, 2003

[8] J.P. Den Hartog, Mechanical Vibrations, fourth edition, McGraw-Hill, New York, 1956. 